\title{
EL PAPEL DE LA ADMINISTRACIÓN LOCAL EN EL DESARROLLO DE LOS ESTÍMULOS FINANCIEROS A LA PEQUEÑA EMPRESA
}

\author{
THE ROLE OF LOCAL ADMINISTRATION IN THE \\ DEVELOPMENT OF FINANCIAL STIMULATIONS TO SMALL \\ BUSINESSES
}

\author{
Alba PERALES GONZÁLEZ ${ }^{1}$
}

Técnica de Administración local, España

Recibido: 05 Abril 2021 / Aceptado: 12 Abril 2021

\begin{abstract}
Resumen
La Administración local goza de una situación privilegiada respecto a otras administraciones como la autonómica o la estatal. Su ubicación territorial le proporciona una proximidad que le permite palpar de primera mano las necesidades y demandas de sus empresas, sus agentes económicos y de la sociedad civil en general. Los estímulos financieros que un gobierno local emplea a través de sus políticas monetarias y fiscales influyen de manera importante en la actividad económica de un municipio. En este artículo repasamos las diferentes políticas que siguen los ayuntamientos para incentivar a la pequeña empresa.
\end{abstract}

Palabras clave: Pymes, Políticas locales, Beneficios fiscales, Subvenciones, Incentivos, Tributos, Grupos de interés, Asociacionismo, Emprendedor

\begin{abstract}
The local administration enjoys a privileged situation compared to other administrations such as the regional or state administrations. Its territorial location provides it with a proximity that allows it to feel first-hand the needs and demands of its companies, its economic agents and civil society in general. The financial stimuli that a local government uses through its monetary and fiscal policies have an important influence on the economic activity of a municipality. In this article we review the different policies that municipalities follow to encourage small businesses.
\end{abstract}

Keywords: SMBs, Local policies, Tax benefits, Subsidies, Incentives, Taxes, Interest groups, Associations, Entrepreneur 
SUMARIO: I. Introducción. II. La relación de las pequeñas empresas con el modelo económico de España. III. La globalización plantea nuevos retos. IV. Incidencia de la política de la UE sobre las Pymes. V. La llegada de la Pandemia COVID-19. VI. Ayudas e incentivos dirigidos a la pequeña empresa. VII. El poder de las Corporaciones Locales. VIII. Estímulos financieros para la pequeña empresa liderados por la Administración Local. IX. La influencia de los grupos de interés y el asociacionismo. X. Conclusiones. XI. Bibliografía.

SUMMARY: I. Introduction. II. The relationship of small companies with the economic model of Spain. III. Globalization poses new challenges. IV. Impact of EU policy on SMEs. V. The arrival of the COVID-19 Pandemic. VI. Aid and incentives aimed at small businesses. VII. The power of Local Corporations. VIII. Financial stimuli for small businesses led by the Local Administration. IX. The influence of interest groups and associations. X. Conclusions. XI. Bibliography.

\section{INTRODUCCIÓN}

El ordenamiento jurídico español establece como Administración Pública una pluralidad de administraciones:2 la Administración General del Estado, las administraciones de las Comunidades Autónomas, las entidades que integran la Administración local y el sector público institucional.

España se puede considerar como uno de los estados más descentralizados a escala mundial. Nos encontramos delante de un estado descentralizado que cede sus competencias en algunos temas, otorgando autonomía a sus comunidades autónomas (MARTÍNEZ-VÁZQUEZ, 2014, pp. 1-34) y éstas a su vez a un nivel más inferior a las administraciones locales.

Las Comunidades Autónomas de España se regulan a través de sus 17 estatutos de autonomías y los dos estatutos de las ciudades de Ceuta y Melilla, y a nivel local existen 8.131 municipios.

Las entidades locales son un conjunto heterogéneo formado por provincias, entidades locales superiores al municipio (comarcas, áreas metropolitanas y mancomunidades), municipios y entidades locales más pequeñas que los municipios pero que las incluyen, siendo por excelencia el municipio la entidad local básica de la organización territorial del Estado. ${ }^{3}$

El presente artículo se centra en la Administración local que existe en los más de 8.100 municipios que configuran el territorio español. Esta gestión es asumida bien a través de las propias sedes de los ayuntamientos o bien a través 
de las juntas de distrito, o de otras oficinas locales en ciudades de tamaño medio y grande. Nos focalizaremos en este caso en los instrumentos que se utilizan para estimular a la pequeña empresa a través de los ayuntamientos.

Los municipios gozan de personalidad jurídica plena, siendo el ayuntamiento quien tiene asignada la función de gobernar, y su administración se produce a través de sus alcaldes y los concejales como regulan el art. 140 y ss de la CE (Constitución Española de 1978). En consecuencia, la Administración local goza de autonomía administrativa y financiera, aunque a un nivel menor que las comunidades autónomas, permitiéndole aprobar diferentes normas en el ámbito de sus competencias como son, entre otras, las ordenanzas generales, las ordenanzas fiscales, los reglamentos o subvenciones.

En diferentes artículos de la CE (art.103.1, 150.3, 155...) se regula que el interés general en las Administraciones Públicas sea un principio de actuación legitimador.

La cercanía con la ciudadanía permite a la Administración local ser un buen canalizador de peticiones y demandas, sin olvidar la influencia de diferentes colectivos económicos, culturales, sociales, educativos, etc.

\section{LA RELACIÓN DE LAS PEQUEÑAS EMPRESAS CON EL MODELO ECONÓMICO DE ESPAÑA}

En primer lugar, tomaremos de referencia la terminología que utiliza el Tribunal de Justicia de la Unión Europea en sus reiteradas sentencias, ${ }^{4}$ en las que define la empresa como "toda entidad, independientemente de su forma jurídica, que ejerza una actividad económica". De esta definición se desprende que lo que importa es la actividad económica y no la forma jurídica entre las posibles.

A su vez, en el ámbito fiscal se diferencia entre actividad profesional y actividad empresarial. ${ }^{5}$ Cuando la actividad es ejercida en una organización (academia, clínica, comercial, etc.) se considera actividad empresarial mientras que se considerará actividad profesional cuando el sujeto realiza directa y personalmente la actividad.

En segundo lugar, considero que es importante fijarnos en la definición de Pyme. ${ }^{6}$ Es necesario para adquirir la condición de Pyme que la entidad sea considerada primero empresa. Por consiguiente, dependiendo de los criterios de personal (Unidades de Trabajo Anual, UTA) y del volumen de negocios (o balance general anual), los siguientes umbrales nos determinaran si se incluyen o no como Pymes: 
- El primer umbral de 10 efectivos $^{7}$ no se puede sobrepasar en microempresas, 50 en pequeñas empresas y 250 en medianas empresas.

- El segundo umbral es el volumen de negocios anual o su balance general anual en millones de euros no puede ascender más de 2 en microempresas, 10 en empresas pequeñas y 50 en empresas medianas.

La Comisión Europea editó una guía muy completa dónde se recoge de forma muy detallada la definición de este concepto, la Pyme, ${ }^{8}$ e incluso ha puesto a disposición una herramienta digital que realiza la evaluación a través de las preguntas de un cuestionario que permite determinar si una empresa es Pyme o no. ${ }^{9}$

Cada estado cuenta con su propia definición del término Pyme. Una de las posibles categorizaciones es basarse en el número de empleados. Como decíamos, España considera que una entidad es Pyme si cuenta con un número de efectivos (generalmente serán asalariados) que esté por debajo de 250.

En tercer lugar, atendiendo a una recomendación de la Comisión Europea, se puede desglosar el concepto de Pyme en tres categorías: microempresas, pequeñas empresas y medianas empresas.

En cuarto lugar, revisando el Directorio Central de Empresas ${ }^{10}$ (DIRCE) a fecha 1 de enero de 2019, éste refleja que existen un total de 3.363.197 empresas activas en territorio español. El 99,93\% de las empresas son Pymes según su criterio de tener un máximo de 200 trabajadores, y el resto, un $0,17 \%$ de empresas, cuentan con un volumen superior a 200 trabajadores. Además, desglosando este valor se observa que el $95,5 \%$ de las empresas son microempresas. Si tenemos en cuenta el criterio de la UE son microempresas aquellas que cuentan hasta de 9 asalariados, son un total de 3.213.557 empresas. En total, el desglose son 1.882.745 empresas sin asalariados, 905.013 empresas con hasta 2 trabajadores, 302.375 con entre 3 y 5 trabajadores, 202.817 con entre 6 y 19 trabajadores, y 64.365 con entre 20 y 199 trabajadores.

Es necesario recalcar que, si nos fijamos en la explotación estadística de la evolución de las Pymes durante las últimas dos décadas, desde el 1999 hasta el 2019, se observa que la tendencia es bastante estable y mantenida (MINGORANCE-ARNÁIZ, 2016, pp. 7-22) con los valores desglosados en el anterior párrafo. Todas estas observaciones se relacionan también con que las actividades económicas en territorio español se dan principalmente en el sector terciario, también llamado sector servicios. 
En general, este sector abarca las actividades que no implican la producción de bienes materiales, por ej, actividad comercial, servicios tecnológicos, servicios profesionales, actividades financieras o turismo y hotelería entre otros. $^{11}$ El sector servicios representa al sector con más del $75 \%$ de su población empleada, el valor en la industria es del $21 \%$, mientras en que en agricultura es del $4 \%$.

\section{LA GLOBALIZACIÓN PLANTEA NUEVOS RETOS}

La globalización económica que vivimos ha permitido a los países operar fuera de sus límites físicos territoriales, abriendo sus fronteras que tantas limitaciones comerciales representaban, y con ello aportando tanto beneficios como dificultades. Se establecen unas nuevas reglas del juego (NELSON, 2018). Esto ha conllevado la internacionalización y expansión de los mercados, de los trabajadores y un cambio dinámico de las interacciones que se producen en este nuevo mundo globalizado.

Si tenemos en cuenta este proceso de globalización como uno un proceso dinámico de interdependencia entre diferentes estados, las empresas deben adaptarse a este nuevo entorno para que el mercado no las excluya (GUTIÉRREZ, 2017, pp. 79-88).

Para que todo el proceso de comunicación digital fluya se debe contar con buenas infraestructuras en telecomunicaciones, así como con todo el operativo para la comunicación digital, en el que un puntal clave es tener a la gente bien formada en nuevas tecnologías, para que dispongan de suficiente madurez en las Tecnologías de la Información y de la Comunicación (TICS).

En España, un reto para muchas microempresas según datos del 2019 es que no existe la suficiente cobertura y despliegue de fibra óptica. En el territorio español, esta es del $80 \%$ como se aprecia a través de la consulta de las zonas blancas y las zonas grises. ${ }^{12}$ Para un estado donde la mayoría de actividad comercial no se encuentra todavía digitalizada, esta limitación supone un importante reto.

\section{INCIDENCIA DE LA POLÍTICA DE LA UE SOBRE LAS PYMES ${ }^{13}$}

En general, en cada estado miembro de la UE las Pymes operan a escala nacional, y son la columna vertebral de la economía europea.

La Unión Europea (UE) establece diferentes planes de acción en su Agenda $2030,{ }^{14}$ los cuales tendrán su afectación también en el mundo local. Son las instituciones europeas las que desarrollan de forma conjunta los objetivos 
políticos de la UE, siendo la Comisión Europea la que despliega la estrategia marcada, transformándola en políticas e iniciativas concretas.

Existen fondos que la UE gestiona directamente, como son subvenciones y contratos que se adjudican mediante el sistema de licitación. Estas subvenciones son para proyectos relacionados con las políticas de la UE.

La estrategia Europa 2020 supone fondos que se gestionan de forma compartida por la UE con las administraciones nacionales y regionales. Los principios de esta estrategia se han basado en el crecimiento inteligente, sostenible e integrador. $\mathrm{Y}$ en general se aplica a través de los siguientes cinco instrumentos:

- El Fondo Europeo de Desarrollo Regional (FEDER), pensado para el desarrollo regional y urbano, prestando especial atención a las características específicas de cada región como pueden ser zonas ultraperiféricas o zonas desfavorecidas geográficamente.

- El Fondo Social Europeo (FSE), que pretende la inclusión social y una buena gobernanza.

- El Fondo de Cohesión (FC), enfocado a la convergencia económica de aquellas regiones que se encuentran menos desarrolladas.

- El Fondo Europeo Agrícola de Desarrollo Rural (FEADER) y el Fondo Europeo Marítimo y de Pesca (FEMP), que van dirigidos al sector primario principalmente.

- El Fondo de Solidaridad de la UE, pensado como respuesta a grandes catástrofes naturales como pueden ser incendios, terremotos, tormentas, (...).

La Agenda 2030, que se aprobó en el 2015, persigue el objetivo de desarrollo sostenible (en adelante ODS). Su hoja de ruta incluye 17 objetivos para el ODS. Entre estos, el objetivo número 8 es trabajo decente y crecimiento económico, el número 9 es industria, innovación e infraestructura, el número 11 ciudades y comunidades sostenibles, y el número 12 es producción y consumo responsable.

La UE se adapta a la era digital, y en este sentido establece como una de las seis prioridades para 2019-2024 una Europa adaptada a la era digital. ${ }^{15}$ Esta prioridad se basa en reforzar la estrategia digital que persigue que esta transformación funcione para las empresas y personas, de cualquier medida y sector para que puedan competir en igualdad de condiciones y desarrollarse, comercializar y usar la tecnología para aumentar su productividad y competitividad mundial. 
Para el período 2019-2024 la UE ha marcado seis prioridades:

1. Un pacto verde europeo (economía sostenible)

2. Una economía al servicio de las personas y las pequeñas empresas

3. Una Europa adaptada a la era digital

4. Promoción de nuestro modo de vida europeo

5. Una Europa más fuerte en el mundo (reforzar nuestro liderazgo mundial responsable)

6. Un nuevo impulso a la democracia europea

Una de las peticiones que más reiteran los empresarios es mejorar su financiación, por un lado, ampliando la concesión de financiación por parte de las entidades financieras. En este sentido, ${ }^{16}$ las formas más usuales de financiación que ofrece la UE son los préstamos, las garantías y las subvenciones.

En cuanto a los préstamos, estos se engloban en diferentes programas tal como Cosme, InnovFin, Europa Creativa (dirigido al sector cultural y creativo) o el programa para el Empleo y la Innovación Social (EaSI) con micropréstamos de hasta $25.000 €$.

Otra parte son las garantías, que corresponden básicamente a la microfinanciación e inversiones de capital riesgo/de capital privado, como es el caso del Banco Europeo de Inversiones y el Fondo Europeo de Inversiones.

La última parte son las subvenciones que corresponden a ayudas directas.

Cabe destacar que el Acelerador del Consejo Europeo de Innovación es otro instrumento que ofrece financiación y mentoría a las Pymes de carácter innovador.

Los operadores internacionales (Fondo Europeo de Inversiones, Banco Europeo, (...) son facilitadores de acceso al crédito a las microempresas, otorgando ayudas económicas en los diferentes estados, aunque a veces estas no se reparten de forma equilibrada y no llegan a las empresas más jóvenes según el informe de la Confederación Española Jóvenes Empresarios (CEAJE) del año $2020 .^{17}$

\section{LA LLEGADA DE LA PANDEMIA COVID-19}

Si se mira con perspectiva, se proclamó el Estado de Alarma por la crisis sanitaria COVID-19, con un confinamiento general ordenado por el Gobierno Central que comportaba el cierre de toda actividad económica que no fuera esencial. Posteriormente se han ido flexibilizando las medidas, y desde hace 
un tiempo son las Comunidades Autónomas quienes deciden, asesoradas por su grupo de expertos.

El confinamiento se ha producido a diferentes niveles territoriales, en períodos de tiempo diferentes y con distintas mediadas. Cada Comunidad Autónoma ha establecido sus prioridades, sus limitaciones y restricciones para evitar la movilidad de las personas y con ello la propagación del virus.

De forma súbita las compras de los productos cuotidianos alimentarios se han visto en algunos casos remplazadas por un servicio de entrega en el domicilio por parte de un proveedor que está ubicado fuera del municipio. Las reglas del juego han cambiado.

Los gigantes del comercio electrónico como Amazon y Aliexpress, con su complejo sistema de venta a través del cual comercializan productos de vendedores a través de Internet, han venido con fuerza para quedarse, sabiendo captar las necesidades de sus clientes: entrega inmediata, una amplia variedad y especialización de productos, conocer las opiniones de otros compradores antes de comprar el producto, etc.

Todo ello tiene una afectación directa en la ciudadanía, y una repercusión muy directa en las actividades económicas de los municipios, con especial atención a sus establecimientos comerciales, mercados, mercadillos y ferias. Se ha instaurado la cita previa para acceder a ciertos servicios y esto habilita una nueva dinámica que se tendrá que ver si y como se mantiene una vez resuelta esta situación pandémica.

La pandemia ha acelerado el acercamiento por parte de las empresas a las nuevas tecnologías. La necesidad de vender de los comercios que están cerrados o bien limitados en su horario comercial ha acelerado su entrada en el mundo digital de forma abrupta y acelerada, de manera que han emergido nuevos canales de venta: pedidos por mensajería instantánea como el whatsapp o recogiendo pedidos por teléfono, nuevas páginas web, pedidos distribuidos a través de plataformas ya consolidadas como Amazon, Uber, Globo o Deliveroo entre otras. Algunos de los comerciantes y empresarios han contado con la suficiente madurez digital, pero ¿y el resto?

En los municipios con una alta fuga comercial a municipios vecinos (pues sus habitantes prefieren los comercios de otros municipios) el confinamiento municipal ha provocado que se evitará temporalmente tal fuga.

Teniendo en cuenta que en muchas empresas se ha instaurado el teletrabajo como modo preferente, esto ha incidido en que los servicios de reformas y ventas de ciertos electrodomésticos, equipamiento para la casa y la oficina 
han experimentado un ascenso, mientras que otros sectores, como agencias de viaje, ocio y cultura, se han desplomado.

España cuenta dos modelos comerciales ben diferenciados. Por un lado, el de Madrid y por el otro el de Barcelona. ¿Cómo se podrá ayudar a los comercios locales para que mantengan su actividad y no se ven obligados al cierre?

El modelo de Barcelona es un modelo de comercio de proximidad, de centros comerciales abiertos (CASTRESANA, 1999, 35-42), menos delimitado, pero más extenso. Los centros comerciales abiertos han sido una fórmula que ha logrado con éxito la modernización y revitalización de los centros urbanos, como por ejemplo en Andalucía. ${ }^{18}$

El modelo de Madrid es un modelo con un centro muy dinámico (Centro y Salamanca) pero con una periferia poco activa, delimitándose un centro comercial muy claro y en el que en el resto de los distritos decae su dinamismo comercial.

\section{AYUDAS E INCENTIVOS DIRIGIDOS A LAS PYMES}

Las diferentes Administraciones Públicas españolas cuentan en sus presupuestos con partidas destinadas a la creación, promoción e internacionalización de la Pyme.

A nivel Estatal, los presupuestos generales del Estados, que son de carácter anual, muestran una previsión de la relación de ingresos y gastos que se van a producir en España. A partir de la pandemia COVID-19 se han reajustado los presupuestos, priorizando la sanidad y salvar la economía de España, a través de unos presupuestos atípicos para el ejercicio 2021. ${ }^{19}$ La dotación presupuestaria se clasifica a través de diferentes programas como por ejemplo el programa $430^{a}$ Comercio, Turismo y PYMES- Mecanismo de Recuperación y Resiliencia o el programa 433M Apoyo a la pequeña y mediana empresa, entre otros.

A nivel Autonómico y local se repite la misma dinámica, pues se aprueban unos presupuestos anuales con la previsión de ingresos y gastos que tendrá para ejecutar las líneas estratégicas que se definan, pudiendo llegar a existir una duplicidad de ayudas que ofrecen las diferentes administraciones (CCAAs, ayuntamientos, diputaciones).

Otro instrumento bastante utilizado es el PAE. El Punto de Atención al Emprendedor facilita que se puedan crear nuevas empresas, el inicio efectivo de su actividad económica y su desarrollo. Existen diferentes puntos 
de atención al emprendedor que dependen tanto de entidades públicas (ayuntamientos, diputaciones...) como de privadas, cámaras de comercio, o colegios profesionales.

\section{EL PODER DE LAS CORPORACIONES LOCALES}

Cada ayuntamiento establece sus planes estratégicos y los proyectos/ actuaciones que deben darse para llegar hasta el objetivo marcado por su equipo de gobierno. Las políticas locales están marcadas por el equipo de gobierno, y dependerá de éstos que se apliquen unas u otras, como pueden ser unas rebajas impositivas o generar una línea de subvenciones para ayudar a un determinado colectivo.

La organización del territorio de un municipio es fundamental para favorecer el crecimiento económico, fomentando una distribución equilibrada del crecimiento para poder alcanzar niveles de renda adecuados en todo el territorio, así como dar un crecimiento ordenado de las implantaciones para hacer más eficaces las actividades económicas y mejorar la calidad de vida. Con carácter general, la ordenación del territorio ${ }^{20}$ es un pilar muy importante para un municipio y su ayuntamiento, aunque técnicamente la competencia es finalmente de las CCAA. Es el marco jurídico general el que da coherencia a una vasta serie de políticas específicas, y se le reconoce superior jerarquía al planeamiento territorial sobre el urbanístico.

Dentro de un territorio se debe establecer la ordenación adecuada de las implantaciones comerciales para poder alcanzar un nivel de equipamiento comercial equilibrado, entre las diferentes formas de distribución y satisfacer las necesidades de compra de los consumidores.

El equipamiento comercial en el ámbito urbano se implanta de dos maneras bien diferenciadas: las que se ubican en el núcleo urbano (acostumbra a ser el casco histórico del municipio) y las que se sitúan en espacios periféricos respecto el centro.

Dependiendo del municipio puede darse que el equipamiento comercial en su conjunto sea insuficiente o incluso inexistente en algunas zonas del municipio. El comercio de proximidad se basa en la variedad de equipamiento comercial y de productos de consumo cuotidiano. Este tipo de comercio debe servir para reducir la movilidad, evitar los desplazamientos innecesarios, generar puestos de trabajo, proporcionar seguridad, cohesión social, establecer más relaciones sociales, mejorar el servicio de los ciudadanos y contribuir a la sostenibilidad de los municipios. 
Las actuaciones que se llevan a cabo para impulsar y mejorar el comercio urbano por parte de ayuntamientos, asociaciones de comerciantes, y cámaras de comercio son conocidas con el nombre de Planes de Dinamización Comercial. La finalidad principal de estos instrumentos es ayudar a los establecimientos comerciales a que desarrollen su actividad en el marco del comercio urbano, y a competir de una manera más eficiente en un entorno tan dinámico como es el actual.

Los planes de dinamización comercial buscan crear e impulsar una opción de compra organizada en un territorio concreto y que sea entendida por el consumidor final. Esta proximidad permite a la ciudadanía, empresarios, agentes sociales, y sociedad civil en general interactuar de algún modo u otro con el equipo de gobierno. A veces, se produce una estrecha relación con la administración local, ya sea de forma presencial o telemática, en la que la administración local es participe de recomendaciones y sugerencias a comerciantes y empresarios, así como recoger nuevas propuestas de acciones.

La cercanía de las empresas con la administración local permite generar unas interactuaciones que no se producen en otras administraciones. Cada municipio se rige por su propio presupuesto municipal, sus ordenanzas municipales y por unas normativas específicas de cumplimiento obligado según el tipo de actividad que se desarrolle. Es en el presupuesto municipal dónde se detallan los ingresos y los gastos previstos para el ejercicio (un año).

Existen municipios que regulan en sus ordenanzas municipales la restricción de actividades en una zona, que pueden frenar la promoción/creación de nuevas empresas. Pero en el sentido contrario de la balanza también existen los premios, las subvenciones y ayudas públicas municipales que conceden los ayuntamientos en su plan de mandato (por norma general abarca 4 años) y sus diferentes líneas estratégicas, que pueden incluir la reactivación económica después de la pandemia.

Las políticas que se pueden seguir en un municipio pueden generalmente ser catalogadas como políticas fiscales expansivas o bien contractivas, pudiendo elevar gastos o bien reducir ingresos (ya sea rebajando impuestos o bien suprimiéndolos). Dependiendo de la política fiscal que se adopte se producirá un mayor o menor beneficio fiscal (LOPEZ ESPADAFOR, 2017, pp. 9-18).

\section{ESTÍMULOS FINANCIEROS PARA LA PEQUEÑA EMPRESA LIDERADOS POR LA ADMINISTRACIÓN LOCAL}

Las siguientes herramientas de las que dispone un ayuntamiento se encuentran ordenadas según su envergadura, de forma general. 


\section{INSTRUMENTO DE PLANTEAMIENTO GENERAL}

La principal razón de que este instrumento esté en primera posición es su carácter transversal y la imposibilidad de llevar a cabo cualquier actuación que no se ciña al Planteamiento General del municipio.

El marco normativo de este instrumento recae en exclusiva a las Comunidades Autónomas debiéndose tener en cuenta su normativa específica respecto al contenido y al alcance de este instrumento de planteamiento general. Las implicaciones de esta herramienta inciden de lleno en la política económica. Con el paso del tiempo, las necesidades y las prioridades de los diferentes territorios se van alterando a nivel urbanístico, siendo necesario su actualización. Si miramos hacia atrás, para ver como era un mismo municipio hace 30-40 años, nos daremos cuenta de que han cambiado muchísimo (nuevas avenidas, nuevas zonas peatonales, nuevas rotondas, el centro de la ciudad puede haberse desplazado, $(\ldots))$.

Cada municipio se rige por su Plan General de Ordenación Urbana (PGOU). Este plan se debe actualizar para poder satisfacer el interés general del municipio. Se plantean nuevas necesidades y se deben adaptar con un nuevo PGOU, tramitando uno de nuevo. Los Ayuntamientos se rigen por sus planes generales de ordenación urbana (PGOU), aunque a veces pueden denominarse de otras maneras. Por ejemplo, en Cataluña éste equivale al Plan de Ordenación Urbanística Municipal (POUM), en Galicia al Plan Xeral de Ordenación Municipal (PXOM) o en Castilla La Mancha Plan de Ordenación Municipal (POM), aunque la esencia es la misma. Cada territorio necesita disponer de esta ordenación territorial, que debe ir ajustándose al modelo territorial que se ha diseñado para ese territorio, en definitiva, al modelo de ciudad que se persigue alcanzar.

Este instrumento de planteamiento urbanístico clasifica el suelo en las siguientes tipologías: suelo urbano, suelo urbanizable y suelo no urbanizable, así como su calificación (uso y posibilidades edificatorias). Existen diferentes herramientas de consulta como son los mapas urbanísticos de las CCAA, por ejemplo, el Mapa Urbanístico de Cataluña de la Generalitat ${ }^{21}$ (MUC) o bien la sede electrónica del Catastro. ${ }^{22}$

\section{PLAN DE BARRIOS}

Es una actuación que seda en una zona determinada de una ciudad, normalmente para revertir las desigualdades existentes entre barrios de la ciudad. La inversión económica puede llegar a ser muy elevada (de millones de 
euros), con la que se mejoran barrios, áreas urbanas y villas que requieran una atención especial. Cada CCAA legisla sobre este tema, siendo el municipio el beneficiario de este plan, por ejemplo, en Gijón, ${ }^{23}$ en las que se aportaron los instrumentos necesarios para realizar intervenciones de carácter social y urbanístico.

Este plan de barrios pretende girar la situación del barrio dotándolo de buenas infraestructuras y equipamientos, como pueden ser escuelas, instalaciones deportivas, arreglos de parques, jardines, arreglos en aceras y pavimentos entre otras acciones. Se intenta con un plan de barrios buscar un mayor equilibrio en la ciudad.

\section{PEATONALIZACIÓN DE LOS CENTROS URBANOS}

Con este tipo de actuación se persigue hacer más transitable un centro urbano, revitalizando la zona, dotándola de mobiliario urbano, y a menudo produciéndose un cambio de imagen considerable. La prioridad se le da al peatón y no al vehículo. Este nuevo entorno provoca que la gente pasee y con ello aumente la actividad comercial, hostelera y de servicios. Normalmente este incremento de actividad comporta una mayor interacción social. Sin embargo, las peatonalizaciones tienen sus virtudes y sus defectos (ALDUÁN, 2014, pp. 67-70). El resultado de la peatonalización casi siempre es positivo, y después nadie quiere volver a la situación anterior. Como, por ejemplo, la peatonalización del centro de Jaén que se llevó a cabo en 2017.

\section{4. ÁREAS DE PROMOCIÓN ECONÓMICAS URBANAS (APEUS)/BIDS}

Una ciertamente nueva figura de promoción y mejora de las áreas urbanas que se inspira en el modelo de éxito de los Business Improvement Districts (BIDs) empieza a tomar fuerza (MITCHELL, 2001, pp. 115-123). Recientemente en Cataluña ${ }^{24}$ se ha aprobado la Ley de las APEUS (BRIFFAULT, 1999, pp. 365-477). Esta herramienta de promoción y mejora de la competitividad de las áreas económicas se basa en la colaboración público-privada. En resumidas cuentas, en un municipio se crea una asociación gestionada entre entidades públicas y privadas a través de la cual todos los negocios de un área definida geográficamente y durante un tiempo limitado se le incrementará sus impuestos a cambio de poder disfrutar de mejoras anteriormente pactadas en la zona. 
5. CELEBRACIÓN DE FERIAS, MERCADILLOS, RUTAS GASTRONÓMICAS, CONCIERTOS Y SIMILARES

La celebración en un municipio de ferias, mercadillos, rutas de las tapas y otros eventos similares que concentren gran cantidad de público puede ser todo un reclamo para el comercio y la restauración del municipio. Este tipo de eventos requieren que se deba valorar los pros y los contras que supone por parte del consistorio en cuestión. Ejemplos de estos eventos son Fitur en Madrid, el descenso del Sella (Ribadesella), un concierto anual de los 40 principales (Rubí) o el Salón del Manga (Barcelona).

\section{VIVEROS DE EMPRESA}

También se conocen con el nombre de incubadoras de empresas o de negocios. Cada vez se han ofrecido más espacios físicos destinados a la promoción de las iniciativas emergentes emprendedoras que combinan la utilización de despachos con el asesoramiento en la gestión empresarial y la prestación de unos servicios comunes generalmente durante los primeros años de su constitución. Si nos basamos en el estudio realizado por la Fundación de Cajas de Ahorro, entre el 70 y el $90 \%$ de las empresas nacidas en un vivero continúan con la actividad después de dejar el vivero (FERNÁNDEZ, 2014, pp. 53-62).

\section{VENTANILLA ÚNICA EMPRESARIAL (VUE)}

La mayoría de los ayuntamientos disponen de este servicio, sobre todo aquellos que tienen una población de más de 50.000 habitantes. Desde 2015 se han integrado las VUEs con los Puntos de Atención al Emprendedor ( PAE $^{25}$ ). Este servicio actúa como ventanilla única para todas aquellas personas que quieren emprender, empresas, comercios e intermediarios que necesitan realizar consultas y trámites relacionados con el entorno empresarial.

\section{CENSO DE ACTIVIDADES ECONÓMICAS DEL MUNICIPIO}

Conocer el número de actividades empresariales que existen en un municipio es básico para poder determinar su evolución, realizar comparaciones entre años, así como dar a conocer a la ciudadanía esta información. Un ejemplo de ello lo encontramos en el Ayuntamiento de Jaén, que realizará en breve este censo en comercios y hostelería. ${ }^{26}$ Acostumbra a pasar que se realiza un censo por parte del ayuntamiento, pero esta fotografía queda rápidamente desfasada debido al dinamismo de las actividades del sector comercial (apertura, cambios 
y cierres de tiendas), por lo que es siempre importante irla actualizando en la medida de las posibilidades.

\section{DIRECTORIO DE EMPRESAS Y COMERCIOS}

Disponer de esta herramienta, así como aplicaciones para dispositivos móviles que permiten la localización de éstos y su difusión, permite a la ciudadanía encontrar empresas y comercios del municipio a través de Internet y redes sociales. Ejemplos de ello los encontramos en la nueva app de la Diputación de Barcelona "Apropteu"27 o el directorio de comercios del Ayuntamiento de Gavà.

\section{CREACIÓN Y DESARROLLO DE UNA MARCA DE CIUDAD}

La creación de una marca de ciudad se basa en un valor intangible de la reputación de una ciudad. Se consigue a través de sus productos, servicios o mensajes que sean lo suficientemente atractivos y que cubran las necesidades de los clientes. Este valor tiene la capacidad de atraer inversores privados y sobre todo, se da a nivel turístico. Por ejemplo, la marca turística de la ciudad de Jaén es "Jaén, Jaén. Auténtica".

\section{ELIMINACIÓN DE TASAS MUNICIPALES}

En general, los ayuntamientos reciben ingresos por dos tipos de tasas. Por un lado, aquellas que se engloban con la prestación de servicios individualizados en el contribuyente, y por otro por el ejercicio del poder de la policía administrativa.

Todas aquellas tasas que se decida que no se van a cobrar al contribuyente en un municipio deberán ser eliminadas de las cuotas establecidas en sus ordenanzas municipales. Está permitido modificar las ordenanzas municipales para no cobrarlas en un futuro, pero si están vigentes las tasas se deben cobrar hasta su anulación. Normalmente se eliminan tasas municipales para poder beneficiar a un sector determinado de la población fundamentándose en diferentes razones, como pueden ser económicas en el caso de la restauración con la tasa de ocupación de las terrazas por parte de bares y restaurantes.

\section{ELIMINACIÓN DE TRIBUTOS POTESTATIVOS}

Hay tributos indirectos que son de aplicación voluntaria, ${ }^{28}$ como el impuesto de construcciones, instalaciones y obras, también denominado (ICIO) y el 
impuesto sobre el Incremento del Valor de los Terrenos de Naturaleza Urbana (IIVT).

En el primero, en el ICIO, al existir una mayor demanda de construcción en los municipios mayores (con mayor número de habitantes), los tipos impositivos se triplican o multiplican, siendo los tipos impositivos más altos los que superan el 3,7\%.

En el segundo, el IIVT, también conocido como plusvalía municipal, se grava un incremento del valor de los terrenos de naturaleza urbana propiedad del contribuyente. Solo se debe pagar este impuesto en el momento de su venta o donación. La plusvalía no debe exceder del 30\%.

\section{EXENCIÓN DEL IMPUESTO DE ACTIVIDADES ECONÓMICAS (IAE)}

Queda regulada en el art. 35.4 de la Ley 58/2003, de 17 de diciembre, General Tributaria en los siguientes 5 casos:

a) Durante los dos primeros períodos impositivos de aquellos sujetos que inicien su actividad en territorio español (art. $82.1 \mathrm{~b}$ ).

b) Los sujetos pasivos del art. $82.1 \mathrm{c}$ de la ley Reguladora de Haciendas Locales.

c) Las personas físicas.

d) Los sujetos pasivos del IS, Sociedad Civil y entidades sin personalidad jurídica que constituyan una unidad económica o patrimonio separado susceptible de imposición (art. 35.4 LGT), que tenga un importe neto de la cifra de negocios inferior a 1 millón de euros.

e) Los contribuyentes de IRNR que operen en España con EP que tengan un importe neto inferior a 1 millón de euros.

\section{BONOS-COMERCIO}

Se han promovido sobre todo durante el ejercicio 2020, a partir de la pandemia COVID-19. Con los bonos-comercio, los ayuntamientos buscan nuevas medidas para revitalizar el sector comercial. La corporación municipal asigna una partida presupuestaria, por ejemplo, de 25.000 euros, en bonoscomercio para incentivar las compras, y de esta manera reactivarla economía de sus comercios, como han realizado los Ayuntamientos de Andújar o de Baena. $^{29}$ 


\section{SUBVENCIONES EN EL ÁMBITO LOCAL}

La mayoría de los consistorios ofrecen algún tipo de subvención a sus ciudadanos y empresas, existiendo dos tipos de procedimiento de concesión de subvenciones: la concurrencia competitiva y las subvenciones directas.

El primero, el de la concurrencia competitiva, es el procedimiento ordinario que se rige a través de compararse las solicitudes presentadas de acuerdo con los criterios que se han fijado anteriormente en unas bases reguladoras, así como en la convocatoria de la subvención. ${ }^{30}$ Es común seguir el orden de entrada por el registro de una solicitud, y si se cumplen los requisitos se irá otorgando hasta finalizar el crédito presupuestario asignado. Ejemplo de ello son las ayudas "Re-activa Autónomos COVID-19" que ofreció el Ayuntamiento de Linares. ${ }^{31}$

El segundo, el de las subvenciones directas, también conocidas como subvenciones nominativas, se establece en dos casos diferentes: unas las que ya se encuentran previstas en los presupuestos generales de la CCAA o si bien impuesto por una norma de rango legal, y las otras son cuando se acredita la dificultad de convocatoria pública o existan razones excepcionales de interés público o económico que lo desaconsejen.

Es habitual establecer convenios con las asociaciones de comerciantes, y a través de estos convenios se ofrecen subvenciones que serán nominativas.

\section{FORMACIÓN Y ASESORAMIENTO}

En muchas ocasiones los ayuntamientos ofrecen asesoramiento a las empresas. Es un servicio específico tanto para la creación de empresas como para su consolidación. Un ejemplo de ello es el Ayuntamiento de Jaén, que dispone de servicios como la elaboración del plan de empresa y análisis de su viabilidad, así como formación empresarial o información sobre ayudas y subvenciones, o la reorientación del negocio.

\section{REEMPRESA}

Existe en algunos municipios de Cataluña el proyecto Reempresa, ${ }^{32}$ un nuevo modelo de emprendimiento empresarial. Consiste en un intercambio profesional por el cual un emprendedor accede a la propiedad de una empresa de otros, en funcionamiento, para hacerse cargo de ella, con la intención de hacerla crecer sin haber de empezar por la fase de creación. En este mecanismo se ponen en contacto la empresa que quiere ceder el negocio y la que lo quiere emprender. 


\section{APOYO A LA BÚSQUEDA DE FINANCIACIÓN}

Se informa de la posibilidad de facilitar información sobre diferentes líneas de subvención, como pueden ser microcréditos (créditos cuyo importe es inferior a 25.000 euros).

\section{PALACIOS FERIALES Y CENTROS DE EXPOSICIONES}

Los municipios que cuentan con más de 200.000 habitantes son los que casi siempre disponen de un equipamiento municipal destinado a ser el aparador de la actividad económica de la zona, y tiene un papel destacado en la dinamización económica y comercial. Por ejemplo, Sevilla o Granada (en el barrio de Almanjáyar) cuentan con recinto ferial, o incluso Manresa (un municipio con 75.000 habitantes en Barcelona) cuenta con el Expobages, un palacio ferial multisectorial de referencia en la Cataluña central.

\section{CESIÓN DE ESPACIOS MUNICIPALES}

Cada vez más se apuesta por ofrecer este tipo de apoyo a las empresas tanto de nueva creación como para las ya existentes. Es una cesión gratuita de espacios de negocio que en general se rige a través de un convenio regulador. Por ejemplo, el IMEFE (Instituto Municipal de Empleo y Formación Empresarial del Jaén) ofrece este servicio.

\section{PATROCINIO}

Los patrocinios y las colaboraciones son otras ayudas que se pueden dar a nivel municipal para fomentar y dar apoyo al tejido empresarial. Normalmente van más destinados a los actos deportivos, pero también se van abriendo camino en rutas gastronómicas, como en la ruta "De tapas por Huelva".

\section{DIFUSIÓN DE OTRAS SUBVENCIONES CON AFECTACIÓN MUNICIPAL}

Es común que los ayuntamientos sean los altavoces de otras ayudas que empresas y comercios pueden solicitar de otras administraciones o entidades. Un ejemplo de ello es la convocatoria de subvenciones para proyectos de ahorro, eficiencia energética, fomento de las energías renovables y reducción de emisiones de $\mathrm{CO} 2$ que informa el Ayuntamiento de Aguilar de la Frontera sobre la Agencia Provincial de la Energía. ${ }^{33}$ 


\section{OTRAS HERRAMIENTAS}

En inglés se utiliza el término crowfunding (PALOMO ZURDO, 2017) para designar una modalidad de financiación que generalmente se instrumentaliza a través de las Plataformas de Financiación Participativa (PFP). Ésta es una modalidad más que tienen las empresas que complementa a la financiación tradicional. En ella se pueden distinguir 4 modalidades:

a) Financiación Propia Participativa (los que aportan fondos pasan a ser socios o accionistas de la empresa).

b) Financiación Ajena Participativa (los que aportan fondos o proyectos financiados pasan a ser inversores-acreedores

c) Crowdfunding de donación

d) Crowdfunding de recompensa

\section{LA INFLUENCIA DE LOS GRUPOS DE INTERÉS Y EL ASOCIACIONISMO}

La participación de diferentes agentes económicos, sociales y de la formación del territorio deben permitir consensuar las políticas de desarrollo económico local.

El poder que tienen las asociaciones de comerciantes que actúan en representación de un colectivo a veces numeroso (con más de un centenar de socios) le legitima y le da un poder para negociar políticas que no es nada despreciable.

Podemos dividir esta participación en foros, consejos y mesas especializadas.

\section{LOS FOROS DE EMPRESAS Y COMERCIOS}

A partir de la pandemia COVID-19 se ha producido un aumento significativo de los foros que repasan el entorno tanto macroeconómico como microeconómico, donde un tema que se ha abordado de forma reiterada son las medidas de apoyo en el proceso de digitalización de una empresa. Estas conferencias o debates son eventos que se difunden habitualmente en streaming, y posteriormente permiten visualizarse en diferido a través de Internet. 


\section{LOS CONSEJOS}

Estos consejos acostumbran a ser órganos permanentes de participación sectorial de carácter consultivo con las finalidades de información, propuesta, consulta y asesoramiento en el desarrollo de políticas e iniciativas que afectan al sector designado (por ej. comercial). Se rigen a través de su propio reglamento y se convocan de forma periódica. Ejemplo de ellos son el Consejo de Comercio de Rubí o el Consejo Sectorial de Comercio de la ciudad de Málaga.

Cuentan habitualmente con la participación de los representantes municipales, técnicos y del equipo de gobierno, las asociaciones de comerciantes de la ciudad, comerciantes no asociados, así como entidades representativas del trabajo autónomo como puede ser la Unión de Profesionales y Trabajadores Autónomos ${ }^{34}$ (UPTA).

Dependiendo del municipio, éste optará facilitar la participación ciudadana a través de diferentes procesos para tomar decisiones sobre el municipio como pueden ser la rehabilitación de una plaza, o la asignación presupuestaria de una parte de su presupuesto, que no suele superar el 5\% de su importe total.

Por ejemplo, en Córdoba se distinguen 3 órganos de participación ciudadana: ${ }^{35}$

- Consejos de Distrito. Es el órgano permanente de participación que extiende sus funciones en un territorio básico, el distrito, para tratar los asuntos de cualquier sector que afecte a dicho territorio y que analiza y coordina las actuaciones que afectan a su territorio.

- Consejos sectoriales. Son los órganos de participación que canalizan las iniciativas e inquietudes ciudadanas en temas concretos de interés para la ciudad como, por ejemplo, la escuela, el deporte, el medio ambiente, la juventud, las mujeres, la gente mayor, las personas con disminución, (...)

- Consejo del Movimiento Ciudadano. Es el órgano permanente de participación que extiende sus funciones a toda la ciudad, para tratar los asuntos de cualquier sector y que analiza y coordina las actuaciones que afectan a su territorio.

\section{LAS MESAS ESPECIALIZADAS}

En estas mesas se puede debatir la planificación estratégica de promoción económica y gobierno. Ejemplos de ello son la mesa de turismo de Valladolid tal como se encuentra reflejado en su Plan Estratégico de Turismo de la ciudad 
de Valladolid 2016-2019, o la Taula de la Competitivitat Industrial de Rubí (TCIR), un órgano de concertación y gobernanza con los agentes de interés del territorio, empresa y conocimiento.

\section{CONCLUSIONES}

El peso que tienen las Pymes en España es muy importante, gozando de un tratamiento fiscal aventajado (BARRUSO, 2016, 100-113) igual que en otros países de la UE, tanto a nivel de impuestos directos (IRPF y IS) como de impuestos indirectos (IVA). En España, de los tres sectores económicos sin duda la actividad principal se produce en el sector terciario o de servicios.

Una empresa estará condicionada si tiene la categoría de Pyme o no a la hora de pedir financiación, ya sea financiación directa o bien financiación indirecta, y a la hora de solicitar ayudas y subvenciones, a cualquier nivel, tanto europeo, estatal, autonómico o local. De aquí la importancia de si una empresa goza la condición de Pyme o no.

Como hemos visto, los gobiernos locales tienen atribuidas por ley ciertas competencias como son la gestión y ordenación del territorio o el urbanismo, según establece en general el Estatuto de Autonomía de cada comunidad autónoma. También tienen la potestad de establecer algunos impuestos y tasas, como son el IIVTNU, el ICIO o las tasas por la ocupación de terrazas en la vía pública.

Las políticas locales que lleve a cabo cada ayuntamiento vendrán definidas por una multiplicidad de variables (BRUGUÉ, 1998, pp. 257-268): su territorio, la singularidad del municipio, la evolución de su población, sus infraestructuras, su actividad económica entre otras, así como por los procesos participativos que operen en el municipio.

Dependerá de las líneas estratégicas que siga un ayuntamiento que este adopte unas políticas, unos programas y proyectos. Los estímulos serán aquellas acciones que tienen el propósito de generar algunas reacciones positivas. Normalmente los estímulos se encuentran vinculados a las políticas monetarias y fiscales que influirán de lleno en la actividad económica.

Estas políticas influirán en futuras decisiones, como pueden ser la rebaja de determinados tributos locales que pueden ser modificados (exenciones o bonificaciones) para liberarlos total o en parte de su carga económica, o la generación de nuevas líneas de subvenciones destinadas a Pymes para que digitalicen su negocio y ganen en competitividad, según marca la UE. 
De la misma manera que la actividad comercial necesita contar con algún instrumento de planeamiento territorial, la participación de las asociaciones de comerciantes, las patronales y los grupos de interés juegan un papel decisivo en las actuaciones de la administración local. Su implicación incide en las políticas locales, así como en la asignación de subvenciones a nivel municipal.

\section{BIBLIOGRAFÍA}

ALDUÁN, A. S. (2014), "Elogio y censura de la peatonalización de los centros históricos", Boletín CF+S, (28), pp. 67-70, http://polired.upm.es/index.php /boletincfs/article/view/2851/2912, [consultado 21 de marzo de 2021].

BARRUSO CASTILLO, B. (2016), La tributación de las pymes en España. Núm. 149 Colegio de Economistas de Madrid, pp. 100-113, https://www.ce mad.es/wp-content/uploads/2016/11/0149.pdf, [consultado 21 de marzo de 2021].

BRIFFAULT, R. (1999), "A Government for Our Time--Business Improvement Districts and Urban Governance", Colum. L. Rev., vol. $99, \mathrm{n}^{\mathrm{o}} 365, \mathrm{pp}$. 365-477, https://scholarship.law.columbia.ed $\mathrm{u} /$ cgi/viewcontent.cgi?article=1017\&context=faculty_scholarship, https:// doi.org/10.2307/1123583, [consultado 21 de marzo de 2021].

BRUGUÉ, J. y GOMÀ CARMONA, R. (1998), Gobiernos locales y políticas públicas: bienestar social, promoción económica y territorio, Ariel Ciencia Política, Barcelona, https://dialnet.unirioja.es/servlet/libro?codigo= 1302, [consultado 21 de marzo de 2021].

CASTRESANA SANCHEZ, J (1999), Nuevas concepciones del urbanismo comercial. Los centros comerciales abiertos, https://dialnet.unirioja.es/serv let/articulo?codigo=168825, [consultado 21 de marzo de 2021].

FERNÁNDEZ KRANZ, D. (2014), Wage adjustment in Spain during the economic crisis, https://www.funcas.es/wp-content/uploads/Migracion/Arti culos/FUNCAS_SEFO/013art07.pdf, [consultado 30 de marzo de 2021].

GUTIÉRREZ, G. B. (2017), "Ciudad digital: paradigma de la globalización urbana". Bitácora Urbano Territorial, 27(1), pp.79-88, http s://revistas.unal.edu.co/index.php/bitacora/article/view/51349/html, https:// doi.org/10.15446/bitacora.v27n1.51349, [consultado 30 de marzo de 2021].

LÓPEZ ESPADAFOR, C. M. (2017), Privilegios e inmunidades fiscales, Tirant lo Blanch, Valencia, https://editorial.tirant.com/es/libro/privilegios-einmunidades-fiscales-carlos-maria-lopez-espadafor-9788491199472, [consultado 21 de marzo de 2021]. 
MARTÍNEZ-VAZQUEZ, J. (2014), "La Descentralización Tributaria a las Comunidades Autónomas en España: Desafíos y soluciones", en Duran, J. y Esteller-Moré, A. (Eds.), Por una verdadera reforma fiscal, Editorial Ariel, pp. 1-34.

MINGORANCE-ARNÁIZ, A. C. y PAMPILLON OLMEDO, R. (2016), La Evolución de las pymes en España, no 149, Colegio de Economistas de Madrid, https://www.cemad.es/wp-content/uploads/2016/11/0149.pdf [consultado 30 de marzo de 2021].

MITCHELL, J. (2001), "Business improvement districts and the 'new' revitalization of downtown", Economic Development Quarterly, 15(2), pp. 115-123 https://journals.sagepub.com/doi/pdf/10.1177/0891242401015002 01, https://doi.org/10.1177/089124240101500201, [consultado 30 de marzo de 2021].

NELSON, D. R. (2018), La nueva economía y política de la globalización, https://www.bbvaopenmind.com/wp-content/uploads/2018/ 03/BBVA-OpenMind-Douglas-R-Nelson-La-nueva-economia-y-politica-d e-la-globalizacion.pdf, [consultado el 30 de marzo de 2021].

PALOMO ZURDO, R. J. (2017), La inteligencia colectiva y la financiación participativa o crowdfunding, https://dictumabogados.com/e-dictum-public aciones-derecho-mercantil/la-inteligencia-colectiva-la-financiacion-partici pativa-crowdfunding/16663/, [consultado el 30 de marzo de 2021].

NOTAS

1 Técnica de Administración Local. Correo electrónico: apg@ajrubi.cat.

2 Art. 2.3 de la Ley 40/2015, de 2 de octubre, de Régimen Jurídico del Sector Público, que regula el ámbito subjetivo de la misma.

3 En su título II sobre el municipio de la Ley 7/1985, de 2 de abril, Reguladora de las Bases del Régimen Local

4 Sentencia del Tribunal de Justicia de 27 de febrero de 2014. http://curia.europa.eu/juris/docum ent/document.jsf?docid=148389\&doclang=ES

5 http://www.ipyme.org/es-ES/EjercicioAct/OFiscales/Paginas/IRPF.aspx\#7

6 La definición de PYME se encuentra recogida en el Anexo I del Reglamento (UE) no 651/2014 de la Comisión Europea

7 Art. 2 Recomendación 2003/361/CE de la Comisión, sobre la definición de microempresas, pequeñas y medianas empresas. https://ec.europa.eu/docsroom/documents/15582/attachments/ $1 /$ translations/es/renditions/pdf

8 https://www.boe.es/buscar/doc.php?id=DOUE-L-2014-81403

9 http://soypyme.ipyme.org/

10 https://www.ine.es/jaxiT3/Datos.htm?t=299\#!tabs-tabla

11 Porcentaje de población empleada en los diferentes sectores económicos de España de 2007 a 2019 https://es.statista.com/estadisticas/501606/distribucion-empleo-en-espana-por-sectoreseconomicos/ 
12 https://avancedigital.maps.arcgis.com/apps/webappviewer/index.html?id=1f63f6fc84b443dc9 $96346 \mathrm{c} 5 \mathrm{e} 32657 \mathrm{~b} 8$

13 https://www.europarl.europa.eu/factsheets/es/sheet/63/las-pequenas-y-medianas-empresas

14 https://www.un.org/sustainabledevelopment/es/development-agenda/

$15 \mathrm{https}$ ://ec.europa.eu/digital-single-market/en/content/european-digital-strategy

$16 \mathrm{https} / /$ europa.eu/youreurope/business/finance-funding/getting-funding/access-finance/index es.htm

17 https://www.ceaje.es/medidas-economicas-ceaje/

$18 \mathrm{https} / / / \mathrm{www}$.europapress.es/esandalucia/cadiz/noticia-centros-comerciales-abiertos-formula-e xito-modernizacion-revitalizacion-centros-urbanos-20180711185008.html

19 Ley 11/2020, de 30 de diciembre, Presupuestos Generales del Estado para el año 2021 https:/ /www.boe.es/eli/es/1/2020/12/30/11

20 Definición recogida en la Carta Europea de la Ordenación del Territorio de 1983.

21 http://ptop.gencat.cat/muc-visor/AppJava/home.do

22 http://www.sedecatastro.gob.es/

23 https://www.elcomercio.es/gijon/plan-barrios-gijon-ana-gonzalez-20191029155229-nt.html

24 Ley 15/2020, de 22 de diciembre, de las áreas de promoción económica urbana. https://www. boe.es/eli/es-ct/1/2020/12/22/15/con

25 Real Decreto 127/2015, de 27 de febrero, por el que se integran los centros de ventanilla única empresarial y la ventanilla única de la Directiva de Servicios en los Puntos de Atención al Emprendedor

26 http://www.aytojaen.es/portal/p_20_contenedor1.jsp?seccion=s_fnot_d4_v1.jsp\&contenido=4 $8111 \&$ tipo $=8 \&$ nivel $=1400 \&$ layout $=$ p_20_contenedor $1 . j \operatorname{sp} \& \operatorname{codResi}=1 \&$ language $=e s \& \operatorname{codM}$ enu $=285 \& \operatorname{cod} M e n u P N=1$

27 https:/www.diba.cat/es/web/comerc/apropteu

28 RDL 2/2004, de 5 de marzo, por el que se aprueba el texto refundido de la Ley Reguladora de las Haciendas Locales (arts. 100 y siguientes).

29 https://www.eldiadecordoba.es/provincia/Baena-repartira-bonos-comercio-incentivar-compras ___1521448287.html

$30 \quad$ LGS art. 22.1

31 https://www.ciudaddelinares.es/ayudas-re-activa-autonomos-covid-19

32 http://reempresa.org

33 https://aguilardelafrontera.es/convocatoria-de-subvenciones-para-proyectos-de-ahorro-eficien cia-energetica-fomento-de-las-energias-renovables-y-reduccion-de-emisiones-de-co2/

34 https://www.upta-andalucia.org/upta-andalucia-se-incorpora-al-consejo-de-comercio-de-mala $\mathrm{ga} /$

35 https://participa.cordoba.es/doc_pdf_etc/documentacion/guia_de_organos_de_participacion.p df 